\title{
Studien über Quercetin und seine Derivate
}

\author{
(VII. Abhandlung) \\ ron
}

Dr. J. Herzig.

Aus dem I. Chemischen Universitätslaboratorium in Wien.

(Vorgelegt in der Sitzung am B. Mai 1891.)

\section{Fisetin.}

Kurze Zeit nach Publication meiner ersten Abhandlungen über das Quercetin hat Schmid ${ }^{1}$ die Untersuchung des Fisetins unternommen und gezeigt, dass die Analysen auf die Formel $\mathrm{C}_{23} \mathrm{H}_{10} \mathrm{O}_{9}$ stimmen und dass das Fisetin anf diese Formel sechs nachweisbare Hydroxyle besitzt. Mit der alten $\mathrm{L}_{i}$ e berm a n n'schen Formel fiir das Quercetin $\mathrm{C}_{24} \mathrm{H}_{16} \mathrm{O}_{11}$ verglichen, weist die Formel fiir das Fisetin einen Mindergehalt von $\mathrm{CO}_{2}$ auf, während die Reactionen dahin zu deuten sind, dass das Quercetin ein Dioxyfisetin sei. Aber nicht allein diese sich widersprechenden Thatsachen haben mich bewogen, das Fisetin in den Kreis meiner Untersuchungen aufzunehmen, sondern auch ganz besonders die merkwïrdige Erscheinung, dass im Fisetin nach Schmid alle Hydroxyle ebenso leicht durch Acetylirung, als wie durch die Alkylirung nachweisbar sein sollten. Die Acetylverbindung be. schreibt er als weisse Substanz, während die Alkylderivate gelb sein sollen. Und doch sollen diese gelben Alkylderivate keine weissen Acetylalkylderivate liefern können.

Wenn man an der nahen Beziehung des Fisetins zum Quercetin festhielt, so standen alle diese Beobachtungen in directem Widerspruch mit meinen damaligen Erfahrungen uber das Quer-

1 Beri. Ber., XIX, 1734. 
cetin. Es ist daher sehr begreiflich, dass ich sofort zu meiner Information das Studium des Fisetins begonnen habe. Nachdem nun jetzt einerseits alle Widersprïche gelöst sind und anderseits weder von Schmid selbst, noch überhaupt aus dem Lung e'schen Laboratorium ïber diesen Gegenstand seither eine Publication erschien, nehme ich keinen Anstand, die Resultate meiner Untersuchung hiemit der Öffentlichkeit zu ïbergeben.

Was die Darstellung des Fisetins betrifft, so habe ich dasselbe Ausgangsmaterial benütyt wie Schmid und habe seiner Beschreibung der Darstellungsmethode nur hinzuzufügen, dass im Bleiniederschlag noch immer Fisetin zurïckbleibt, welches durch Zersetzung mit Schwefelwasserstoff wieder gewonnen werden kann. Dabei kann man entweder den Bleiniederschlag in Alkohol suspendiren und das Gas einleiten oder den Niederschlag unter Wasser zersetzen und nach dem Abfiltriren das Schwefelblei mit Alkohol extrahiren. Selbstverständlich ist das so gewonnene Fisetin nicht so rein, wie das ans dem Filtrat erhaltene. Es genügt aber ein zwei- bis dreimaliges Auflösen in Alkohol und Ausfällen mit Wasser, um ein hellgelbes, aschenfreies Präparat von genügender Reinheit zu bekommen.

\section{Acetylfisetin.}

Dasselbe wurde auf gewöhnliche Weise durch Einwirkung von Essigsäureanhydrid und Natriunacetat auf Fisetin erhalten. Es wurde, da es in Alkohol gar zu schwer löslich ist, theils aus Essigäther, theils aus Eisessig umkrystallisirt. In beiden Fällen erbielt ich die Substanz in Form weisser glänzender Nadeln vom constanten Schmelzpunkt $196-199^{\circ}$ (Sch. 200-201). Die Analysen dieser Substanz ergaben folgende Daten:

1. $0.3060 \mathrm{~g}$ bei $100^{\circ}$ getrockneter Substanz gaben $0.6831 \mathrm{~g}$ Kohlensäure und $0.1121 \mathrm{~g}$ Wasser.

2. $0.3578 \mathrm{~g}$ bei $100^{\circ}$ getrockneter Substanz gaben $0.7977 \mathrm{~g}$ Kohlensäure und $0 ; 1300 \mathrm{~g}$ Wasser.

In 100 Theilen:

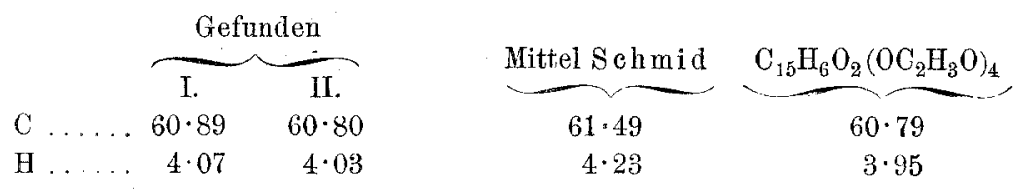


Die Verseifung dieses Acetylderivates wurde nach der bewährten. Methode von Liebermann unternommen. Sie ergab folgendes Resultat:

3. $5740 \mathrm{~g}$ bei $100^{\circ}$ getrockneter Substanz gaben $2 \cdot 2420 \mathrm{~g}$ bei $120^{\circ}$ getrockneten wiedergewonnenen Fisetins.

In 100 Theilen :

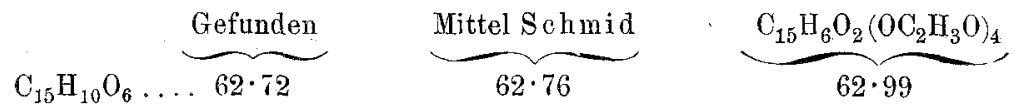

Das auf diese Weise regenerirte Fisetin habe ich wieder analysirt und erhielt dabei folgende Zahlen:

I. $0 \cdot 2931 \mathrm{~g}$ Substanz gaben $0 \cdot 6720 \mathrm{~g}$ Kohlensäure und $0 \cdot 0960 \mathrm{~g}$ Wasser. II. $0.2757 g$ " $\quad 0.6347 \mathrm{~g} \quad$ " $0.0892 \mathrm{~g}$ "

In 100 Theilen:

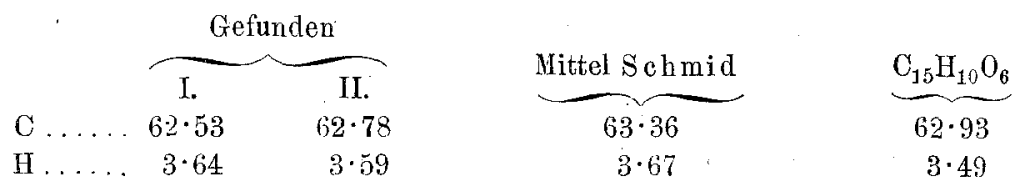

Wie man sieht, stimmen die gefundenen Zahlen ganz gut auf die Formel $\mathrm{C}_{15} \mathrm{H}_{10} \mathrm{O}_{6}$ für das Fisetin. Da nun anderseits im Acetylquercetin fünf Acetoxylgruppen vorhanden sind, während die mitgetheilten Analysen deren nur vier im Acetylfisetin nachweisen lassen, so stehen bei diesen Verbindungen die Thatsachen mit der Auffassung des Quercetins als Oxyfisetin vollkommen im Einklang.

\section{Methylfisetin.}

Interessant gestaltete sich die Untersuchung der Alkylderivate des Fisetins. Schmid's Analysen wiesen darauf hin, dass genau so viele Hydroxylgruppen alkylirt sind, als durch das Acetyliren nachgewiesen werden konnten, was bis zu einem gewissen Grade meinen Beobachtungen beim Quercetin widerstritt. Ich ging an die Untersuchung dieser Verbindungen mit der vorgefassten Meinung, dass hier von Seiten Schmid's ein Versuchsfehler vorliegt. Sind die Substanzen, wie beschrieben, gelb, dann müssten sie noch weisse Acetylalkylderivate liefern und könnten daher unmöglich genau so viele Alkylgruppen 
enthalten, als im Acetylfisetin Acetylgruppen nachweisbar sind. Sollten aber die Analysen von Schmid ganz richtig sein, so müssten die Substanzen jedenfalls weiss erscheinen. Dabei ging ich von der Voraussetzung aus, dass die ron mir damals in der Quercetingruppe angenommenen Chinonsauerstoffe sich beim Fisetin beim Alkyliren genau so anfrollen wie beim Acetyliren. Meine Versuche haben gezeigt, dass die Analysen von Schmid ganz richtig sind, dass man aber beim wiederholten Umkrystallisiren die Alkylderivate des Fisetins absolut weiss ohne jeden Stich ins Gelbe erhalten kann. Allerdings ist die Schwierigkeit, die Substanzen weiss zu erhalten, so gross, dass man diesen Umstand, wenn man nicht direct darauf ausgeht, sehr leicht iibersehen kann.

In Zusammenhang mit meinen Erfahrungen beim Enxantlın und Quercetin erklärt sich dieses merkwïrdige Verhalten ohne jede Schwierigkeit. Von diesem Gesichtspunkte aus ist in Bezug anf diese Reactionen zwischen den drei Körpern gar kein principieller, sondern nur ein gradueller Unterschied vorhanden. Sie lassen sich in eine Gruppe formiren, deren äusserste Glieder Quercetin, respective Fisetin sind, während das Euxanthon eine Mittelstellung einnimmt. Gemeinsam besitzen sie die Eigenschaft, im vollkommen acetylirten oder alkylirten Zustand keine Farbstoffe zu bilden. Diese gemeinsame Eigenschaft wird bedingt durch ein Hydroxyl, welches entweder sich in einer bestimmten Stellung befindet oder in irgend einer Weise einer Umlagerung fähig ist. Während nun beim Quercetin die weissen vollkommen alkylirten Abkömmlinge so zersetzlich sind, dass ich sie bisher nicht erhalten konnte, sind sie umgekehrt beim Fisetin so stabil, dass man auf gewöhnliche Weise nur diese und nicht die gelben in Kali unlöslichen Alkylderivate erhalten kann. Beim Euxanthon liegen die Verhältnisse so, dass man beide Arten von Verbindungen darstellen kann. Es ist nicht ausgeschlossen, ja ich balte es sogar für wahrscheinlich, dass es mir in Zukunft gelingen wird, die noch vorhandenen Lücken in dieser Reihe anszufüllen.

Was nun die Darstellung und Eigenschaften des Methylfisetins betrifft, so habe ich den Angaben von Schmid nur hinzaznfügen, dass dasselbe in langen weissen glänzenden Nadeln krystallisirt, welche den constanten Schmelzpunkt 151-153 
(Schm. 152-153) besitzen. Die Analyse ergab folgendes Resultat:

1. $0 \cdot 3260 \mathrm{~g}$ bei $100^{\circ}$ getrockneter Substanz gaben $0 \cdot 7939 \mathrm{~g}$ Kohlensäure und $0 \cdot 1634 g$ Wasser.

II. $0 \cdot 2817 \mathrm{~g}$ bei $100^{\circ}$ getrockneter Substanz gaben $0 \cdot 6880 \mathrm{~g}$ Koblensäure und $0 \cdot 1378 \mathrm{~g}$ Wasser.

III. $0 \cdot 2490 \mathrm{~g}$ bei $100^{\circ}$ getrockneter Sulsstanz gaben nach Zeișel $0 \cdot 6891 \mathrm{~g}$ Jodsilber.

IV. $0.2122 g$ bei $100^{\circ}$ getrockneter Substanz gaben nach Z e isel $0.5780 y$ Jodsilber.

V. $0.2132 g$ bei $100^{\circ}$ getrockneter Substanz gaben nach Zeisel $0.5816 g$ Jodsilber.

\begin{tabular}{|c|c|c|c|c|c|c|c|}
\hline & \multicolumn{5}{|c|}{ Gefunden } & Mittel & \multirow[b]{2}{*}{$\mathrm{C}_{15} \mathrm{H}_{6} \mathrm{O}_{2}\left(\mathrm{OCH}_{3}\right)_{4}$} \\
\hline & I. & II. & III. & IV. & V. & $\underbrace{\operatorname{sen} \cos i d}$ & \\
\hline $\mathrm{C}$. & $66 \cdot 41$ & $66 \cdot 60$ & - & - & -- & $66 \cdot 82$ & $66 \cdot 66$ \\
\hline & $5 \cdot 57$ & $5 \cdot 43$ & - & - & - & $5 \cdot 39$ & $5 \cdot 26$ \\
\hline $\mathrm{CH}_{3} \mathrm{O}$. & - & - & $36 \cdot 50$ & $35 \cdot 98$ & $35 \cdot 98$ & - & $36 \cdot 28$ \\
\hline
\end{tabular}

\section{Äthylfisetin.}

In Bezug auf Darstellung und Eigenschaften gilt dasselbe wie beim Methylderivat. Krystallisirt aus Alkohol in weissen gliinzenden Nadeln vom Schmelzpunkt 106-108 (Sch. 106 bis 107). Die Analyse lieferte folgende Daten:

I. $0 \cdot 3369 g$ bei $100^{\circ}$ getrockneter Substauz gaben $0 \cdot 8582 g$ Kohlensäure und $0 \cdot 1960 g$ Wasser.

II. $0 \cdot 2663 g$ bei $100^{\circ}$ getrockneter Substanz gaben $0 \cdot 6747 g$ Kohlensäure und $0 \cdot 1556 \mathrm{~g}$ Wasser.

III. $0.2283 g$ bei $100^{\circ}$ getrockneter Substanz gaben nach $Z$ e ise $0.5310 g$ Jodsilber.

IV. $0 \cdot 2755 \mathrm{~g}$ bei $100^{\circ}$ getrockneter Substanz gaben nach Z eis el $0 \cdot 6465 \mathrm{~g}$ Jodsilber.

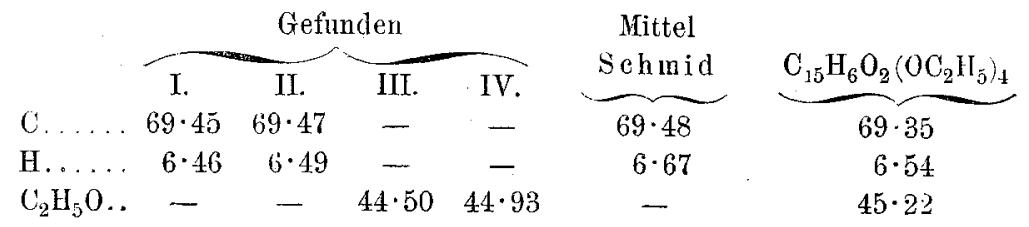

Man sieht aus diesen Analysen, dass die von Schmid und mir gefundenen Zahlen auch bei diesen Fisetinderivaten mit der Thcorie im vollkommenen Einklang stehen. 
Bemerken will ich noch, dass in der That diese weissen Verbindungen, wie schon Schmid hervorhebt, mit Essigsäureanhydrid nicht mehr reagiren, und dass sie daher entschieden keine freie Hydroxylgruppe entbalten können.

Schliesslich möchte ich noch erwähnen, dass ich aus dem Athylproduct zur grösseren Vorsicht mittelst Jodwasserstoffsäure Fisetin regenerirt habe, so dass die Möglichkeit ausgeschlossen ist, dass diese Substanzen nicht mehr Derivate des unzersetzten Fisetins sind.

\section{Oxydation mittelst Luft in alkalischer Lösung.}

Das Fisetin oxydirt sich an der Luft in alkalischer Lösung so leicht wie das Quercetin. Letzteres liefert bekanntlich ${ }^{1}$ bei dieser Gelegenheit Protocatechusäure und Phloroglucin. Wenn nun das Verhältniss beider Substanzen tbatsächlich dabin zu formuliren wäre, dass Quercetin ein Oxyfisetin ist, so muss man bein Fisetin ein um eine Hydroxylgruppe ärmeres Zersetzungsproduct erwarten (Salicylsäure, Paraoxybenzoesäure oder Resorcin). Wäre dies nicht der Fall, so müsste man annehmen, dass im Quercetin eine hydroxylhaltige Seitenkette existirt, die bei der Luftoxydation weg oxydirt wird. Ich habe mich davon überzeugt, dass dies nicht der Fall ist und dass man in der That Protocatechusäure und Resorcin erhält. Fisetin wurde in Kali gelöst und in einer grösseren Schale unter öfterem Umrühren der Einwirkung der Luft ausgesetzt. Nach 24 Stunden ist die Reaction heendet, insofern als sich beim Ansäuern nichts mehr ausscheidet. Die kalische Lösung wurde dann schwach mit Essigsäure angesäuert und mit Bleizucker gefält. Aus dem Bleiniederschlag. konnte eine Substanz erhalten werden, welche alle Reactionen der Protecatechusäure lieferte und den Schmelzpunkt 194-196 ${ }^{\circ}$ besass. Das Filtrat wurde zur Entfernung des Bleies mit Schwefelwasserstoff behandelt, das Schwefelblei abfiltrirt und die Fliissigkeit mit $\ddot{A}$ ther ausgeschïttelt. Der $\ddot{A}$ iher hinterlässt eine krystallinische Substanz, welche durch mehrmalige Destillation gereinigt wurde und in reinem Zustande den Schmelzpunkt 106-108 besass. Auch erhielt ich die Eisen- und die für das Resorcin so charakteristische Fluoresceinreaction.

\footnotetext{
1 Monatshefte für Chemie, VI, 863.
} 
Für die Thatsache, dass Schmid in der Kalisehmelze Protocatechusäure und Phloroglu cin („verhältnissmä ssig wenig“") erhielt, gibt es zwei mögliche Erklärungen. Entweder es liefert Resorcin anch in der Kalischmelze etwas Phloroglucin, worüber ich in der Literatur keine Angabe gefunden habe, ${ }^{1}$ oder Schmid hat die Kalischmelze mit nicht ganz reinem Fisetin unternommen, so dass das Phloroglucin auf Rechnung der Verunreinigungen zu setzen wäre.

Da nun aber umgekehrt dic Bildung von Resorein ans Phlor(glucin bei dieser Reaction höchst unwahrscheinlich ist und ich ausserdem auch bei deu ganz reinen, weissen Alkylderivaten Resorcin erhielt, so ist wohl in meinem Falle ein Irrthum so ziemlich ausgeschlossen. Diese Reaction ist daher als ein weiterer Beweis fur die Auffassung des Quercetins als Oxyfisetin zu betrachten. Dass Schmid kein Resorcin gefunden hat, erklärt sich einfach aus dem Umstand, dass er es, wie es scheint, gar nicht erwartet und daher die Mutterlauge rom Phloroglucin ganz vernachlässigt hat.

\section{Einwirkung von alkoholischem Kali auf Hethylfisetin.}

Mit Riucksicht auf meine diesbezüglichen Versuche bei den Alkylquereetiren war es geboten, diese Reaction auch auf die Alkylfisetine anzuwenden, zumal das entsprechende Resorcinderivat möglicherweise hätte krystallisirt sein können. Als ich das Äthylfisetin mit alkoholischem Kali im Rohr auf $150-160^{\circ}$ erbitzte, zeigte es sich, dass auch hier Diäthylprotocatechusäure entsteht neben cinem Phenol, wclches nicht krystallisirt und von dem nur constatirt werden konnte, dass es die Spanreaction des Pliloroglucins nicht liefert. Die entsprechende Reaction lieferte beim Quercetin ebenfalls Diätlyylprotocatechusäure, aber daneben ein Phenol, welches sich als ein Derivat des Phloroglucins erkennen liess.

Die Zersetzung der Alkylfisetine durch alkoholisches Kali geht aber schon am Rückflusslzühler ganz glatt und quantitativ

1 Teh habe mich inzwischen durch einen Versuch, den Herr stud. Ma yer im hiesigen Laboratorium unternahm, überzeugt, dass reines Resorcin in der Kalischmelze ein rroduct liefert, welches die Spanreaction des Phloroglucins litefert. 
unter Bildung you zwei krystallisirten Verbindungen vor sich, so dass diese Zersetzung wohl einen genïgend sicheren Schluss auf die Constitution des Fisetins zu zieben erlaubt.

Äthylfisetin wurde mit der 10 fachen Menge alkoholischen Kalis am Rückflusskühler am Wasserbade 7 -8 Stunden erhitzt. Nach dieser Zeit wurde der Alkohol abdestillirt und der Kolbeninhalt mit Wasser anfgenommen. Dabei zeigte sich, dass sich Alles bis auf eine geringe Tribung auflöst.

Zur Entfernung dieser Trïbung wurde die Lösung mit $\ddot{\text { Äther }}$ ausgeschüttelt. Der Äther hinterliess immer nur eine Spur einer gelblichen Substanz, welche wogen der geringen Menge nicht weiter untersucht wurde. In die mit Äther ansgeschiittelte Lösung: wurde zur Absättigung des Kalis Kohlensäure bis zur vollständigen Neutralisation eingeleitet, wobei sich eine weisse krystallinische Substanz $(A)$ in beträchtlicher Menge ausscbied. Die Fliissigkeit $(\boldsymbol{B})$ sammt Niederschlag wurde mit Äther ausgeschüttelt, in welchem sich die Substanz sehr leicht löst. Der Äther hinterlässt beim Abdestilliren ein in langen dïnnen Nadeln krystallisirendes Product, welches keine Spanreaction liefert, sich in Kali löst, aber aus dieser Lösung mit Kohlensäure ausfällen lässt.' Sie liefert mit Eisenchlorid eine schwachrothe Färbung, welche auf Zusatz von kohlensauren Alkalien wieder versehwindet. Da die Verbindung in Alkohol zu leicht löslich war, wurde sie aus verdiunntem Alkohol umkrystallisirt. Ich erhielt sie in Form weisser Nadeln rom constanten Schmelzpunkte $42-44^{\circ}$ C. Die Analysen dieser Substanz ergaben folgendes Resultat:

I. $0.3168 g$ im Vacuum über Schwefelsäure getrockneter Substanz gaben $0 \cdot 7456 \mathrm{~g}$ Kohlensäure und $0 \cdot 2050 \mathrm{~g}$ Wasser.

II. $0 \cdot 3051 \mathrm{~g}$ im Vacuum über Schwefelsäure getrockneter Substanz gaben $0.7176 \mathrm{~g}$ Kohlensäure und $0.2000 \mathrm{~g}$ Wasser:

III. $0.2948 g$ im Vacuum über Schwefelsäure getrockneter Substanz gaben nach Z e ise $10 \cdot 6130 \mathrm{~g}$ Jodsilber.

1 I) a sich die Constitution dieser Körper noch nicht vollständig aufklären liess, muss ich vorläufig für dieselben eine provisorische Nomenclatur einführen. Das bei Zersetzung des Methylfisetins entstehende phenolische Produet soll Methylfisetol, das entsprechende Äthylderivat Äthylfisetol heissen. Das alkylfreie Phenol muisste demgemäss bis auf Weiteres Fisetol benannt werden. 
In 100 Theilen:

\begin{tabular}{|c|c|c|c|}
\hline & & refundo & \\
\hline & I. & II. & JII. \\
\hline C... & $64 \cdot 18$ & $64 \cdot 14$ & - \\
\hline $\mathrm{H} \ldots$. & $7 \cdot 18$ & $7 \cdot 28$ & - \\
\hline $\mathrm{C}_{2} \mathrm{H}_{5} \mathrm{O}$ & - & - & $39 \cdot 81$ \\
\hline
\end{tabular}

Diese Zahlen stimmen am besten mit der Formel $\mathrm{C}_{6} \mathrm{H}_{8} \mathrm{O}_{2}$ überein, welche folgende Zahlen verlangt. Auf $\mathrm{C}_{6} \mathrm{H}_{8} \mathrm{O}_{2}$ käme dann $1 \mathrm{C}_{2} \mathrm{H}_{5} \mathrm{O}$.

\begin{tabular}{|c|c|}
\hline $\begin{array}{l}\begin{array}{l}\text { Gefunden } \\
\text { im Mittel }\end{array} \\
\end{array}$ & $\underbrace{\mathrm{C}_{4} \mathrm{H}_{3} \mathrm{O}\left(\mathrm{OC}_{2} \mathrm{H}_{5}\right)}$ \\
\hline $\mathrm{C} \ldots \ldots .64 \cdot 16$ & $64 \cdot 28$ \\
\hline H..... 723 & $7 \cdot 14$ \\
\hline $\mathrm{C}_{2} \mathrm{H}_{5} \mathrm{O} \ldots 39 \cdot 81$ & $40 \cdot 18$ \\
\hline
\end{tabular}

Um über die Moleculargrösse Aufschluss zu erhalten, habe ich versucht, Salze dieser Verbindung mit den Alkalien darzustellen, abel ohne besonderen Erfolg; weil dieselben zu leicht löslich sind und ich nicht viel Substanz zur Verfügung batte. Hingegen gelingt es sehr leicht, mittelst Kali und Jodäthyl den Äthyläther dieses Körpers darzustellen. Derselbe ist in Kali unlöslich and krystallisirt aus verduinntem Alkohol in langen Nadeln, welche constant bei $66-68^{\circ}$ schmelzen. Die Analyse dieses Äthers lieferte folgendes Resultat:

I. $0 \cdot 2361 \mathrm{~g}$ im Vacuum über Schwefelsäure getrockneter Substanz gaben $0.5724 \mathrm{~g}$ Kohlensäure und $0.1738 g$ Wasser.

II. $0.2285 \mathrm{~g}$ im Vacum über Schwefelsäure getrockneter Substanz gaben $0.5560 \mathrm{~g}$ Kohlensäure und $9 \cdot 1676 \mathrm{~g}$ Wasser.

III. $0 \cdot 1687 \mathrm{~g}$ im Vacuum über Schwefelsäure getrockneter Substanz gaben nach Zeisel $0.4678 \mathrm{~g}$ Jodsilber.

In 100 Theilen:

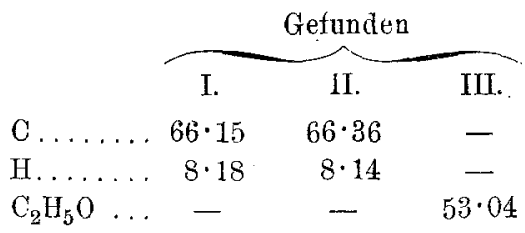

Wie folgende Zusammenstellung zeigt, ist es wahrscheinlich, dass der Äther die Zusammensetzung $\mathrm{C}_{14} \mathrm{H}_{20} \mathrm{O}_{4}$ und nicht 
$\mathrm{C}_{20} \mathrm{H}_{28} \mathrm{O}_{6}$ und es wäre dementsprechend das Äthylfisetol $\mathrm{C}_{12} \mathrm{H}_{16} \mathrm{O}_{4}$ und nicht etwa $\mathrm{C}_{18} \mathrm{H}_{24} \mathrm{O}_{6}$. Das Äthylfisetol wirde daher zwoi Äthoxylgruppen entbalten, während auf dessen Ätbyläther drei Äthoxylgruppen kämen. Dementsprechend wäre dann die Formel des Fisetols $\mathrm{O}_{8} \mathrm{H}_{8} \mathrm{O}_{4}$.

\begin{tabular}{|c|c|c|}
\hline Gefunden & $\underbrace{\mathrm{C}_{8} \mathrm{H}_{5} \mathrm{O}\left(0 \mathrm{C}_{2} \mathrm{H}_{5}\right)_{3}}$ & $\mathrm{C}_{12} \mathrm{H}_{8} \mathrm{O}_{2}\left(O \mathrm{C}_{2} \mathrm{H}_{5}\right)_{4}$ \\
\hline C..... $66 \cdot 26$ & $66 \cdot 67$ & $65 \cdot 93$ \\
\hline H..... $8 \cdot 16$ & $7 \cdot 93$ & $7 \cdot 69$ \\
\hline $\mathrm{C}_{2} \mathrm{H}_{5} \mathrm{O} \ldots 53 \cdot 0 t$ & $53 \cdot 57$ & $49 \cdot 45$ \\
\hline
\end{tabular}

Obwohl die Differenz im Äthoxylgehalt ziemlich bedeutend int war doch Vorsicht geboten, zumal sich bei dieser Verbindung. wieler die Zeis el'sche Bestimmung schwierig gestaltet, weil die Substanz mit Jodwasserstoffsänre verharzt und sich vor Beendigung der Reaction ausscheidet. Man muss daher vorsichtig operiren, möglichst wenig Substanz nelımen und der Jodwasserstoffsäure lissigsätreanhydrid zusetzen.

Wegen dieser Verharzing war ich auch nicht im Stande, las äthoxylfreie Fisetol $\mathrm{C}_{8} \mathrm{H}_{8} \mathrm{O}_{4}$ darzustellen. Das sich ausschcidende harzige Product erinnert sehr lebhatt an das Resorcinharz von Barth und Weidel ind diurfte ihm anch sehr nahe stehen, da, wie ich später zeigen werde, das Äthylfisetol sehr lejelt Resorcin liefert.

Der Äthyläther lässt sich am Rückflusskühler mit allsoholisehem Kali kochen, ohne sich zu verseifen und ist nicht ohne Zersetzung flüchtig.

Wir kommen nun zur Untersuchung der Flissigkeit $(\boldsymbol{B})$, welche nichts weiter als Diäthylprotocatechusäure enthält. Dieselbe wurde durch ihren Schmelzpunkt, Eigensehaften und endlich auch durch die Elementaranalyse als solche erkannt und charakterisirt.

Durch die Oxydation des Fisetins mittelst Sauerstoffes der Luft ist wahrscheinlich gemacht worden, dass im Fisetinmolek ül der Rest der Protocatechnsäure neben dem des Resorcins präformirt enthalten ist. Es war daher vorauszuseher, dass das neben der Diäthylprotocatechusäure entstehende $\ddot{A}$ thylfisetol ein Resorcinderivat sein wird. In der That liefert dasselbe in der 
Kalischmelze ganz leicht Resorcin, welches am Schmelzpunkt und an der Fluoresceïnreaction erkaunt wurde.

\section{Einwirkung von alkoholischem Kali auf Methylfisetin.}

Es wiederholt sich hier Alles genau wie beim Äthylderivat. Das Methylfisetol ist in Alkohol nicht so leicht löslich wie das entsprechende Äthylderivat und wurde daher aus Alkohol umkrystallisirt. Ich erhielt dasselbe in Form kleiner, weisser, kreidiger Nadeln vom constanten Schmelzpunkt 66-68 . Es zeigt mit Eisenchlorid dasselbe Verbalten wie das Äthylderivat. Die Analyse ergab folgendes Resultat:

I. $0 \cdot 3266 \mathrm{~g}$ im Vacuum über Schwefelsäure getrockneter Substanz gaben $0.7301 \mathrm{~g}$ Kohlensäure und $0.1782 \mathrm{~g}$ Wasser.

II. $0.2013 \mathrm{~g} \mathrm{im}$ Vacuum über Schwefelsäure getrockneter Substanz gaben nach $\mathrm{Ze}$ is el $0 \cdot 4789 \mathrm{~g}$ Jodsilber.

In 100 Theilen:

\begin{tabular}{|c|c|c|c|}
\hline \multicolumn{3}{|c|}{ Gefunden } & \multirow[b]{2}{*}{$\mathrm{C}_{4} \mathrm{H}_{3} \mathrm{O}\left(\mathrm{OCH}_{3}\right)$} \\
\hline & I. & II. & \\
\hline $\mathrm{C} \ldots \ldots$ & $60 \cdot 96$ & - & $61 \cdot 22$ \\
\hline $\mathrm{H} \ldots \ldots$ & $6 \cdot 06$ & - & $6 \cdot 12$ \\
\hline $\mathrm{CH}_{3} \mathrm{O} \ldots$ & - & $31 \cdot 63$ & $31 \cdot 37$ \\
\hline
\end{tabular}

Neben diesem Producte wurde, wie zu erwarten war, Dimethylprotocatechusäure nachgewiesen.

Auch hier wurden zur Ermittlung der Moleculargrösse die Äther dieser Verbindung dargestellt und zwar zunächst der

\section{Methyläther des Methylfisetols.}

Über die Darstellung dieses Körpers brauche ich wohl nicht viel zıt sagen, da dieselbe nach der gewöhnlichen Methode ganz leicht gelingt. Er ist in Kali unlöslich, am Rückflusskühler unverseifbar und wurde aus verdunntem Alkohol umkrystallisirt. Er scheidet sich in Form kleiner weisser Nadeln aus, welche den Schmelzpunkt $62-63^{\circ}$ besitzen. Die Analyse lieferte folgende Daten:

T. $0 \cdot 3142 g$ im Vacuum über Schwefelsäure getrockneter Substanz gaben $0 \cdot 7247 \mathrm{~g}$ Kohlensäure und $0 \cdot 1904 \mathrm{~g}$ Wasser.

II. $0 \cdot 1590 \mathrm{~g}$ im Vacuum über Schwefelsäure getrockneter Substanz gaben nach Z eisel $0 \cdot 5265 g$ Jodsilber. 
In 100 'Theilen:

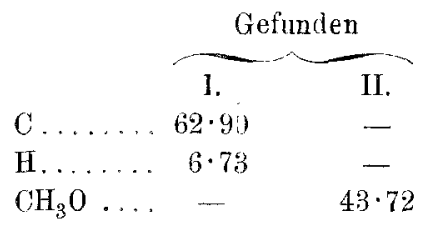

Diese Zahlen stehen in rollem Einklang mit dem oben ewähnten Resultat bei der Analyse des Äthyläthers des Äthyl. fisctols, wie folgende Zusammenstellung zeigen mag:

\begin{tabular}{|c|c|c|}
\hline Gefunden & $\mathrm{C}_{8} \mathrm{H}_{5} \mathrm{O}\left(\mathrm{OCH}_{3}\right)_{3}$ & $\mathrm{C}_{12} \mathrm{H}_{8} \mathrm{O}_{2}\left(\mathrm{OCH}_{3}\right)_{4}$ \\
\hline C . . ... $62 \cdot 90$ & $62 \cdot 86$ & $62 \cdot 33$ \\
\hline H..... $6 \cdot 73$ & $6 \cdot 67$ & $6 \cdot 60$ \\
\hline $\mathrm{CH}_{3} \mathrm{O} \ldots 43 \cdot 72$ & $44 \cdot 28$ & $40 \cdot 2$ \\
\hline
\end{tabular}

Beide Resultate vereint, weisen auf die Formel $\mathrm{C}_{8} \mathrm{H}_{8} \mathrm{O}_{4}$ fiil das Fisetol hin und haben auch das gemeinsam, dass die grössten Differenzen zwischen den von den rerschiedenen möglichen Formeln geforderten Werthen bei der Äthoxyl-, respective Methoxylzahl rorkommen, da die für den Kohlenstoffgehalt berechneten Zahlen zu nahe bei einander liegen. Es ist daher begreif lich, dass ich eine Verbindung gesucht habe, wo die Differenz anch in Kohlenstoffgehalt klar zu Tage tritt und eine derartige Substanz labe ich im

\section{Äthyläther des Methylfisetols}

gefunden. Derselbe wurde bei zwei verschiedenen Darstellungen mit den gleichen Eigenschaften gefunden und krystallisirt aus verdünntem Alkohol in weichen glänzenden Nadeln vom constanten Schmelzpunk $\uparrow$ 60-62 . Die Analyse ergab folgendes Resultat:

Darstellung $A$.

I. $0 \cdot 3041 g$ im Vacuum über $\mathrm{H}_{2} \mathrm{SO}_{4}$ getrockneter Substanz gaben $0 \cdot 7174 g$ Kohlensäure und $0 \cdot 1988 g$ Wasser.

II. $0.3703 \mathrm{~g}$ im Vacuum über $\mathrm{H}_{2} \mathrm{SO}_{4}$ getrockneter Substanz gaben $0 \cdot 8734 \mathrm{~g}$ Kohlensäure und $0.2440 \mathrm{~g}$ Wasser.

Darstellung $B$.

III. $0 \cdot 2835 \mathrm{~g}$ im Vacuum über $\mathrm{H}_{2} \mathrm{SO}_{4}$ getrockneter Substanz gaben $0 \cdot 6662 g$ Kohlensäure und $0 \cdot 1867 \mathrm{~g}$ Wasser.

IV. $0.1898 \mathrm{~g}$ im Vacuum über $\mathrm{H}_{2} \mathrm{SO}_{4}$ getrockneter Substanz gaben nach Z eise $10.5900 \mathrm{~g}$ Jodsilber. 
In 100 Theilen:

\begin{tabular}{|c|c|c|c|c|}
\hline & \multicolumn{4}{|c|}{ Gefunden } \\
\hline & 1. & II. & III. & EV. \\
\hline $\mathrm{C} \ldots$ & $64 \cdot 34$ & $64 \cdot 32$ & $64 \cdot 08$ & - \\
\hline $\mathrm{H}, \ldots \ldots$ & $7 \cdot 25$ & $7 \cdot 31$ & $7 \cdot 2$ & - \\
\hline $\mathrm{C}_{2} \mathrm{H}_{5} \mathrm{O}^{1} \ldots$ & - & - & - & $19 \cdot 37$ \\
\hline
\end{tabular}

Auch diese Analysen stimmen weit besser auf $\mathrm{C}_{8} \mathrm{H}_{5} \mathrm{O}\left(\mathrm{OCH}_{3}\right)_{2}$. . $\left(\mathrm{OC}_{2} \mathrm{H}_{5}\right)$ als auf $\mathrm{C}_{12} \mathrm{H}_{8} \mathrm{O}_{2}\left(\mathrm{OCH}_{3}\right)_{3}\left(\mathrm{OC}_{2} \mathrm{H}_{5}\right)$.

\begin{tabular}{|c|c|c|c|}
\hline & Gefunden & $\mathrm{C}_{8} \mathrm{H}_{5} \mathrm{O}\left(0 \mathrm{CH}_{3}\right)_{2}\left(O \mathrm{C}_{2} \mathrm{H}_{5}\right)$ & $\mathrm{C}_{12} \mathrm{H}_{8} \mathrm{O}_{2}\left(0 \mathrm{CH}_{3}\right)_{3}\left(0 \mathrm{C}_{2} \mathrm{H}_{5}\right)$ \\
\hline $\mathrm{O}$ & $64 \cdot 33$ & $64 \cdot 28$ & $63 \cdot 35$ \\
\hline H... & $.7 \cdot 28$ & $7 \cdot 14$ & $6 \cdot 83$ \\
\hline $\mathrm{C}_{2} \mathrm{H}_{5} \mathrm{O}$ & $19 \cdot 37$ & $20 \cdot 09$ & $13 \cdot 97$ \\
\hline
\end{tabular}

Aus diesen Versuchen kann man folgende für die Constitution des Fisetins wichtige Schlüsse ziehen. Das Fisetin $\mathrm{C}_{15} \mathrm{H}_{10} \mathrm{O}_{6}$ zersetzt sich in seinen Alkylderivaten unter dem Einflusse alkoholischen Kalis im Sinne folgender Gleichung:

$$
\underbrace{\mathrm{C}_{15} \mathrm{H}_{10} \mathrm{O}_{6}}_{\text {Fisetin }}+2 \mathrm{H}_{2} \mathrm{O}=\underbrace{\mathrm{C}_{7} \mathrm{H}_{6} \mathrm{O}_{4}}_{\begin{array}{c}
\text { Proto- } \\
\text { catechusäure }
\end{array}}+\underbrace{\mathrm{C}_{8} \mathrm{H}_{8} \mathrm{O}_{4}}_{\text {Fisetol }}
$$

Mit dieser Gleichung stimmt auch die Menge der erhaltenen Zersetzungsproducte sehr gut überein.

Weiterhin kann man, da alle vier Alkylgruppen des Fisetins in den Zersetzungsproducten wieder auftreten, mit voller Berechtigung schliessen, dass bei dieser Reaction keine Verseifung stattgefunden hat, und es ist dann klar, dass sowohl das in dem thei]weise alkylirten Fisetol vorhandene freie Hydroxyl, als wie die Carboxylgruppe der Protocatechusäure durch Wasseranlagerung entstanden sind.

Was nun die Constitution des Fisetols $\mathrm{C}_{8} \mathrm{H}_{8} \mathrm{O}_{4}$ betrifft, so ist festgestellt, dass dasselbe drei Hydroxyle enthält und dass cs ein Resorcinderivat ist. Diese beiden Thatsachen combinirt, ergeben als Consequenz, dass das dritte Hydroxyl in einer Seitenkette

I Diese Zahl gilt nach Subtraction der für die Methoxylgrippe erforderlichen Menge Jodsilbers. 
sich befinden muss. Ausserdem kann ich schon jetzt mich über die Function des vierten Sanerstoffatoms dahin äussern, dass dasselbe entweder einer Aldehyd- oder Ketongruppe angehören muss, da das Fisetol mit Phenylhydrazin eine Verbindung gibt, die ich seinerzeit beschreiben werde. Obwohl man schon auf Grund dieser Thatsachen eine Constitutionsformel aufstellen könnte, will ich es doch vorläufig unterlassen, da der Körper doch noch nicht genügend studirt ist und alle von mir bisher in Betracht gezogenen Formeln, z. B. $\mathrm{C}_{6} \mathrm{H}_{3}(\mathrm{OH})_{2}, \mathrm{CO} . \mathrm{CH}_{2} \mathrm{OH}$ oder $\mathrm{C}_{6} \mathrm{H}_{3}(\mathrm{OH})_{2}$. CH.OH.COH die leichte Oxydirbarkeit des Fisetols zu Resorcin nicht plausibel machen können. Eine derartige leichte Oxydationsfähigkeit vorausznsetzen, bin ich aber nach meinen bisherigen Erfahrungen absolut genöthigt.

Das genaue Studium dieses Zersetzungsproductes und die richtige Deutung dieser Versuche ist jetat, wie mir scheinen will, zum Kern der Frage nach der Constitution der Körper aus der Qnercetingruppe geworden, da ja das Verhältniss dieser Sub stanzen zum Fisetin so ziemlich klargestellt ist. Da nun die Beschaffung des zu diesem Studium erforderlichen Materials einige Zeit in Anspruch nimmt und ich ausserdem die aus der Enxanthongruppe hergeholten Analogien durch weitere directe Beweise stittzen möchte, darf ich wohl die berechtigte Erwartung aussprechen, dass mir dieses Arbeitsgebiet noch einige Zeit gewahrt bleibt. 